\title{
Work-Related Stress: Lessons From the US Marine Corps
}

\author{
Christopher A. Sinsabaugh MD, Brandon P. Brown MD, MA, Richard B. Gunderman MD, PhD
}

Department of Radiology, Indiana University, Indianapolis, Indiana

Many radiologists find the practice of medicine stressful. As a result, physician disengagement and depersonalization, sometimes addressed as "burnout," have garnered considerable attention of late 1, 2, 3 . Contributors include high personal standards for performance, increasing expectations for productivity, fear of error and its consequences, the rapid pace of change in health care, and innovations in IT. Although in medicine, work-related stress is a relatively new topic of study, other fields have studied it for many years, and their experiences and insights can help illuminate the stress of radiologists. At the forefront of such organizations is the US Marine Corps.

\section{Evolution in Understanding of Work-Related Stress}

The understanding of work-related stress evolved considerably during the 20th century, perhaps nowhere more dramatically than in the Marine Corps [4]. Before World War I, the Marines conceptualized stress injury as the result of physical trauma to the brain, secondary to nearby artillery blasts and the like. After the United States entered the war, the understanding of stress injury shifted from physical damage in the brain to "shell shock," the response of a weak character to severe stress. In managing such cases, the Marine Corps took pains to distinguish between shell-shocked marines and those who were truly medically sick or injured.

Although this model succeeded in reducing the number of stress-related medical evacuations from the theater of battle, it carried with it a number of disadvantages [5]. First, it focused purely on occupational

This is the author's manuscript of the work published in final edited form as:

Sinsabaugh, C. A., Brown, B. P., \& Gunderman, R. B. (2018). Work-Related Stress: Lessons From the US Marine Corps. Journal of the American College of Radiology, 15(3), 493-495. https://doi.org/10.1016/j.jacr.2017.10.024 
health, creating the presumption that if stress-related evacuations were infrequent, the problem was being managed effectively. Second, it placed responsibility for recognition and reporting almost solely on individual marines, contributing to the mistaken assumption that if marines are not complaining, they are fine. Third, and perhaps most important, the character weakness model inevitably contributed to a high level of stigma surrounding shell shock [6]. Subsequent research has shown that preexisting character and personality are far less important than the frequency and intensity of exposure to combat in predicting who will develop the disorder [7].

\section{Current Concept of Work-Related Stress}

Today, the Marine Corps operates with the concept of combat and operational stress, and such problems are referred to as stress injuries. They parallel bodily injuries in a number of respects. First, they increase the risk for other disorders, such as posttraumatic stress disorder, if they do not heal completely. Second, they generally heal more quickly and completely if they are appropriately diagnosed and treated. Third, they often leave scars, which can function as sources of either weakness or strength for the injured.

To clarify the severity of stress, the Marine Corps uses a combat and operational stress continuum model, which features four zones. In the green "ready" zone, the stressed marine is coping well with stress and is ready for duty. In the yellow "reacting" zone, the marine is experiencing mild distress, exhibiting symptoms such as poor focus, disrupted sleep, and lack of enjoyment in work. In the orange "injured" zone, the solider is experiencing more severe distress and may exhibit panic or rage, have recurrent nightmares, or feel guilt and shame. In the red "ill” zone, marines experience loss of function and develop clinical mental disorders such as depression and substance abuse.

Initial evaluations of the Marine Corps' stress control and readiness program have yet to demonstrate improvement in mental health outcomes, perhaps because they are attempting to account for too many variables or because the program is not implemented consistently across all units [8]. However, they have shown that the program increases the rate at which marines seek help with stress-related problems. 
Moreover, members of the Marine Corps consider it a valuable tool for enhancing stress response and recovery efforts.

\section{Responding to Stress}

The Marine Corps now teaches leaders to respond to their service members' work-related stress by focusing on each of five different areas. The first of these is to strengthen individuals, units, and families to enhance their resilience. One means of strengthening is training, which develops physical and mental strength and confidence in abilities. Another is social cohesion, providing marines with shared experiences of accomplishment in overcoming adversity that foster team spirit. Finally, leaders can help marines and their family members stay focused, build confidence, and approach challenges with courage.

A second leadership response to work-related stress is mitigation. The Marine Corps recognizes that no marine is immune to stress and that stress should be kept as much as possible within a manageable range. The Marine Corps conceptualizes resilience and courage in terms of leaky buckets that are constantly being drained by stress. The leader's job is to keep them from running dry by refilling them with sleep, rest, recreation, and spiritual renewal. Moreover, the leader can help prevent more holes from being punched in the bucket.

A third response is identification. Leaders must know their personnel, their strengths and weaknesses, the challenges they face, and how they are responding to stressors. Marines may lose confidence in themselves or others or experience a declining sense of cohesion with comrades. The responsibility for monitoring and responding to such problems rests initially on the shoulders of small-unit leaders, the only individuals who can know their marines and the nature of what they are experiencing sufficiently to recognize and respond to work-related stresses.

A fourth response is treatment. In some situations, individual marines can treat themselves and render aid to one another. In other cases, small-unit leaders or chaplains can make important contributions. And in 
still other cases, professional medical or psychological help may be called for. Often family members can play an important role in helping prevent harm and promote healing from a stress injury. Especially important is the effort to combat the stigma associated with stress injuries, which tends to undercut efforts at diagnosis and treatment across all levels.

The final leadership response to work-related stress is reintegration. In most cases, stress injuries will tend to get better over time, especially if they are appropriately treated. Good mentorship can play an important role in promoting the healing process. Leaders need to recognize not only when their service members are experiencing stress but also when they have recovered sufficiently to be restored to full duty. Also, both the individuals recovering from stress injuries and their peers and leaders need opportunities for the restoration of confidence, a process that cannot happen overnight.

\section{Implications for Radiology}

For radiologists, one implication of the Marine Corps' approach to stress is clear: the problem does not get better by ignoring it. Radiologists and radiology departments need to better understand the nature of physician burnout and work-related stress. This means becoming familiar with its signs and symptoms, the factors that contribute to it, its consequences, and the measures available to combat it. Moreover, stigmatizing the difficulty associated with work-related stress does no one a service. If anything, it tends to drive it underground, allowing its adverse effects to build up to a breaking point, which can have adverse and even catastrophic consequences both for individual radiologists and their colleagues and families.

The Marine Corps clearly states that leadership must create an environment in which work-related stress is recognized, mitigated, and treated. In terms of strengthening radiologists, one of the most promising areas is education. Educators need to ensure that radiology residents and fellows are entrusted with increasing levels of responsibility to help them develop realistic expectations for work life and strategies for handling their own stress. Examples might include escalating expectations for case complexity and 
volumes, increasing clinical independence in on-call duties, and graded responsibility for discussing and responding to errors.

The multiple experience levels and hierarchy inherent in the Marines have parallels in medical training. Just as the infantry includes multiple privates, several corporals, and a sergeant, so a medical team may be composed of several students, an intern and resident, and a faculty physician. Although military training heavily emphasizes interdependence and cooperation among these levels, too often medicine promotes a culture of excessive independence and autonomy among physicians. Radiology leaders and educators need to highlight the importance of teamwork, in which practitioners see themselves as collaborators helping one another toward a larger goal.

Some situations inevitably induce stress, such as the care of critically ill patients and trauma victims. Other sources of stress are avoidable. Examples include inadequate resources (a poor IT system), inefficient work systems (spending hours each day attempting to contact referring physicians), and reducing the concept of physician value to productivity quotas (particularly when this compromises radiologists' sense that they are doing high-quality work and making a difference). The Marine Corps condemns the view that its service members could ever be regarded as "fire and forget" weapons, a lesson that should not be lost on radiologists.

Good leadership means caring not just about throughput and productivity but also about the people doing the work, which in turn means remaining well acquainted with how each radiologist is faring and responding quickly when signs of trouble become apparent. The Marine Corps color-coded stress zones model can be helpful in determining what level of stress each colleague is experiencing. Indicators that a colleague is in serious trouble include symptoms and signs of panic disorders, alcohol or drug abuse, and major depression, which can manifest in unexpected forms such as aggression.

Treatment begins with reducing stigma, including the widespread fear that admitting difficulties will result in career damage. A radiology department or group should not tolerate a culture in which members 
feel compelled to destroy themselves in order to keep up or make themselves sick in order to heal others. In some cases, colleagues need to be encouraged to take more regular breaks or use more of their paid time off. In other cases, the solution lies in enhancing engagement with the work itself, for example by reducing isolation and loneliness.

Finally, radiology departments need to practice reintegration. We once asked a very senior radiologist, a man who had won the top awards of the major radiology organizations, what decision or action over the course of his career brought him the deepest sense of pride and fulfillment. Without hesitation, he answered, "Taking a chance on a radiologist who had been fired twice from other practices due to alcoholism. My colleagues thought I was crazy, but after talking with the man, I became convinced that he deserved another chance." To the radiology leader's delight, the radiologist in question turned out to be a highly effective clinical radiologist who won a number of departmental awards for teaching excellence.

\section{Conclusions}

While lamenting the prevalence of military metaphors to describe the ravages of stress in contemporary radiology practice ("I got killed on call last night"), we believe that the Marine Corps offers important insights on the nature and effective management of work-related stress. It recognizes that psychological health is every bit as important as physical health, and it invests considerable resources in promoting it. Radiology practices need to do the same. The field of radiology and the patients it cares for can thrive only to the extent that radiologists themselves are psychologically healthy and thriving. 


\section{References}

1. McNeeley MF, Perez FA, Chew FS. The emotional wellness of radiology trainees: prevalence and predictors of burnout. Acad Radiol 2013;20:647-55.

2. Gunderman RB, Phadke S. Burnout's up-side. J Am Coll Radiol 2016;13:1538-40.

3. Harolds JA, Parikh JR, Bluth EI, Dutton SC, Recht MP. Burnout of radiologists: fre-quency, risk factors, and remedies: a report of the ACR Commission on Human Resources. J Am Coll Radiol 2016;13:411-6.

4. US Marine Corps. Combat and operational stress control. Washington, District of Columbia: US Department of the Navy; 2010.

5. Shay J. Odysseus in America: combat trauma and the trials of homecoming. New York: Scribner; 2002.

6. $\quad$ Corrigan P. How stigma interferes with mental health care. Am Psychol 2004;59:

614-25.

7. Shalev A, Liberzon I, Marmar C. Post-trau-matic stress disorder. N Engl J Med 2017;376:245969.

8. Vaughan CA, Farmer CM, Breslau J, Burnette C. Evaluation of the Operational Stress Control and Readiness (OSCAR) program. RAND Health Quarterly 2015;5:14. 